\title{
Q-switched erbium-doped fibre laser based on molybdenum disulfide and tungsten disulfide as saturable absorbers
}

\author{
${ }^{1}$ Mohamed K.H., ${ }^{1}$ Hamida B.A., ${ }^{1}$ Sheroz Khan, ${ }^{1}$ Hussein L.A., \\ ${ }^{1}$ Ahmat M.O., ${ }^{2}$ El Ismail, ${ }^{2}$ Kadir N.A.A., ${ }^{2}$ Latif A.A. and ${ }^{2}$ Harun S.W. \\ ${ }^{1}$ Department of Electrical Engineering, Faculty of Engineering, International \\ Islamic University Malaysia (IIUM), Jalan Gombak, 53100 Kuala Lumpur, \\ Malaysia \\ ${ }^{2}$ Department of Electrical Engineering, Faculty of Engineering, University of \\ Malaya, 50603 Kuala Lumpur, Malaysia, e-mail: faatax26@gmail.com, \\ belal@iium.edu.my, swharun@um.edu.my
}

Received: 08.11.2016

\begin{abstract}
A passively Q-switched erbium-doped fibre laser is experimentally implemented, using molybdenum disulfide $\left(\mathrm{MoS}_{2}\right)$ and tungsten disulfide $\left(\mathrm{WS}_{2}\right)$ as saturable absorbers. The results testify that a $\mathrm{WS}_{2}$-based $\mathrm{Q}$-switched fibre laser generates more stable pulse trains, if compared with the $\mathrm{MoS}_{2}$-based saturable absorber. The wavelength region where the Q-switched pulses have been obtained is a so-called C-band region. The central wavelengths are 1559 and $1560 \mathrm{~nm}$ for $\mathrm{MoS}_{2}$ and $\mathrm{WS}_{2}$, respectively.
\end{abstract}

Keywords: Q-switching, erbium-doped fibres, $\mathrm{MoS}_{2}, \mathrm{WS}_{2}$, saturable absorbers

PACS: 42.60.Da, 42.55.Wd, 42.60.Gd

UDC: $535.37+681.7 .068$

\section{Introduction}

Fibre lasers have been developing very rapidly in the last decade. Among these devices, lasers based on erbium-doped fibres (EDFs) are the most investigated [1]. The EDFs represent a valuable gain medium since it can amplify the light in the wavelength region of $1550 \mathrm{~nm}$ needed in many applications. [2]. EDF-based fibre lasers (EDFLs) have received significant attention of researchers because of their benefits in the areas of optical communications and fibre sensor applications [3]. In particular, passively Q-switched EDFLs have drawn much attention due to their potential applications in medicine, remote sensing, biomedical diagnostics, metrology, as well as in the fibre optical sensing and telecommunications mentioned above [2]. Notice that the passive Q-switching technique based on saturable absorbers (SAs) has many advantages if compared with the corresponding active techniques, in particular simplicity, compactness and flexibility $[4,5]$. There are a number of effects and techniques that can be used for providing passive Q-switching. They include nonlinear polarization rotation [6], semiconductor SA mirrors, transition metal-doped bulk crystals, graphene, and single-walled carbon nanotubes [7]. It is worthwhile that fabrication process of the semiconductor SA mirrors is complicated and expensive. On the other hand, although single-walled carbon nanotube-based SAs are simple to fabricate and cost-effective, they reveal low damage thresholds and cannot provide a desired wavelength range of SA without matching diameter-dependent bandgap [8].

In principle, molybdenum and tungsten disulphides can become a real alternative for building SAs. $\mathrm{MoS}_{2}$ and $\mathrm{WS}_{2}$ consist of hexagonal layers of molybdenum or tungsten sandwiched between 
two layers of sulphur. $\mathrm{MoS}_{2}$ and $\mathrm{WS}_{2}$ reveal the indirect bandgaps respectively $1.29 \mathrm{eV}(961 \mathrm{~mm})$ and $1.4 \mathrm{eV}(886 \mathrm{~nm})$, whereas the direct bandgaps of their monolayers increase up to $1.80 \mathrm{eV}$ $(689 \mathrm{~nm})$ and $2.1 \mathrm{eV}(\sim 590 \mathrm{~nm})$, respectively [5, 7]. In this article, we aim to demonstrate a Q-switched EDFL that employs the SAs based on $\mathrm{MoS}_{2}$ and $\mathrm{WS}_{2}$.

\section{Experimental setup}

The experimental setup was built in a small room at our laboratory to avoid any changes in the environment temperature. A cavity was placed at a table, as shown in Fig. 1. The cavity contained six main components: a pump power source, a wave division multiplexor, an EDF, an isolator, a 20:80 coupler, and a SA. The laser used a $2.8 \mathrm{~m}$ long EDF as a gain medium, which was pumped by a $980 \mathrm{~nm}$ laser diode via a 980/1550 $\mathrm{nm}$ wave division multiplexor. A polarization-independent isolator prevented the laser from unwanted feedback propagation.

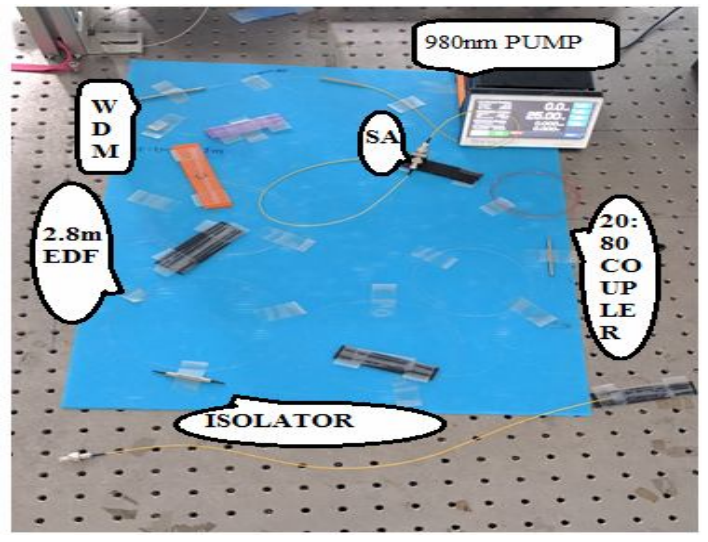

(a)

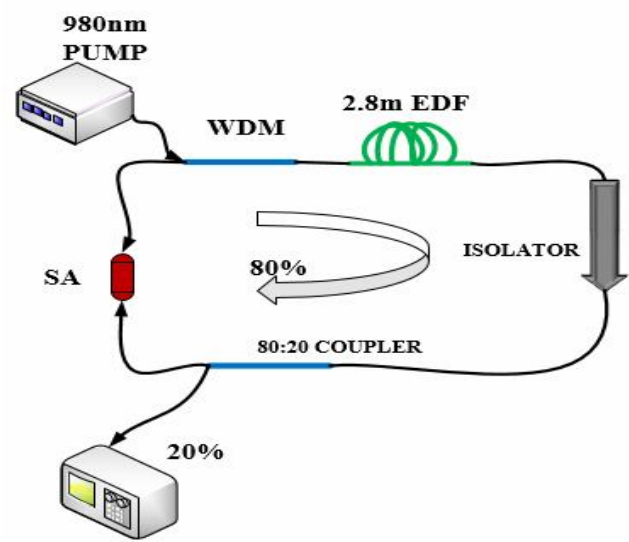

(b)

Fig. 1. A design of our EDFL cavity (a) and its schematic diagram (b).

\subsection{Passively Q-switched fibre laser based on $\mathrm{MoS}_{2}$}

Fig. 2 illustrates the result obtained using a standard OSA device, the output spectrum detected from the passively Q-switched EDFL based on the $\mathrm{MoS}_{2} \mathrm{SA}$, as observed at the pump power

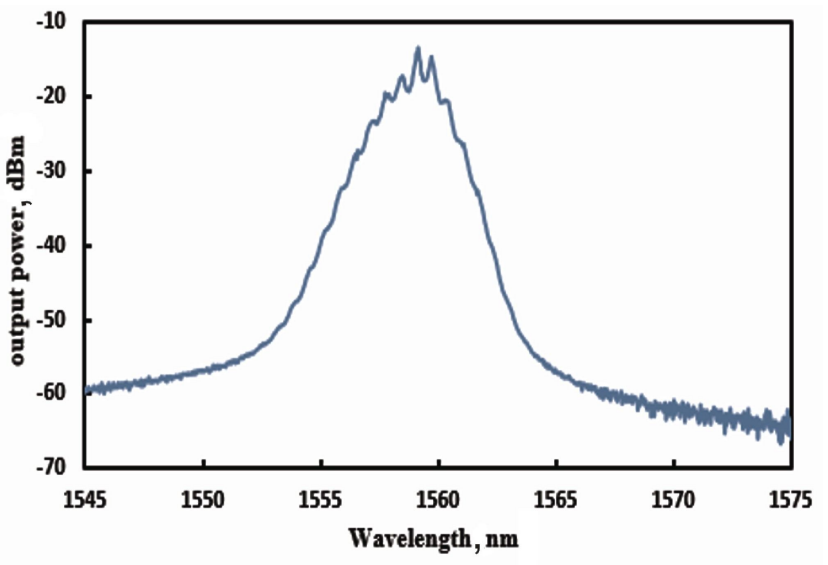

Fig. 2. Output spectrum of our passively Q-switched EDFL based on the $\mathrm{MoS}_{2} \mathrm{SA}$.
$65.7 \mathrm{~mW}$. As seen from Fig. 2, the threshold peak at which our EDFL works corresponds to $1559.16 \mathrm{~nm}$, i.e. to the wavelength region called as a C-band. The spectral bandwidth at $3 \mathrm{~dB}$ is equal to $1.4 \mathrm{~nm}$. Note that the SA plays an important role in our experiment, since it is just that component that ensures obtaining passively Q-switched 
pulses. To confirm the fact that the passive Q-switching is attributed to the $\mathrm{MoS}_{2} \mathrm{SA}$, we have intentionally removed the $\mathrm{MoS}_{2}$ flake from the ring cavity. In this case no Q-switched pulses are observed, even if the pump power is being adjusted over a wide range. On the contrary, if the SA is inserted back into the cavity, the passively Q-switched pulses reappear. Therefore, the SA is strictly responsible for the passive Q-switching in the fibre laser and it is impossible to get the passively Q-switched pulses without the SA.

Fig. 3a illustrates how the average output power of our Q-switched EDFL based on the $\mathrm{MoS}_{2}$ SA depends on the pump power. At the pump powers higher than $20 \mathrm{~mW}$, the average output power of the fibre laser reacts almost linearly to increasing pump power. When the pump power is $116.61 \mathrm{~mW}$, the highest average output power is achieved, which is approximately $5.13 \mathrm{~mW}$. The slope efficiency is then estimated as $4.42 \%$. The dependence of the pulse energy on the pump power is shown in Fig. $3 \mathrm{~b}$. Here the minimum and maximum pump powers are $14.85 \mathrm{~mW}$ to $116.61 \mathrm{~mW}$, respectively. When the pump power reaches its maximal value, the maximal pulse energy, $75 \mathrm{~nJ}$, is achieved. Above this maximal pump power, the Q-switched pulse becomes unstable and can even disappear, which means no Q-switched pulses at all.

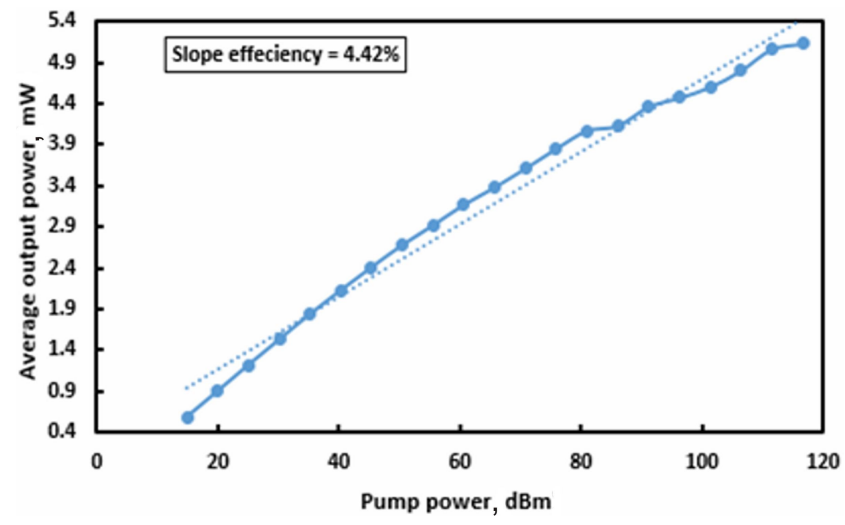

(a)

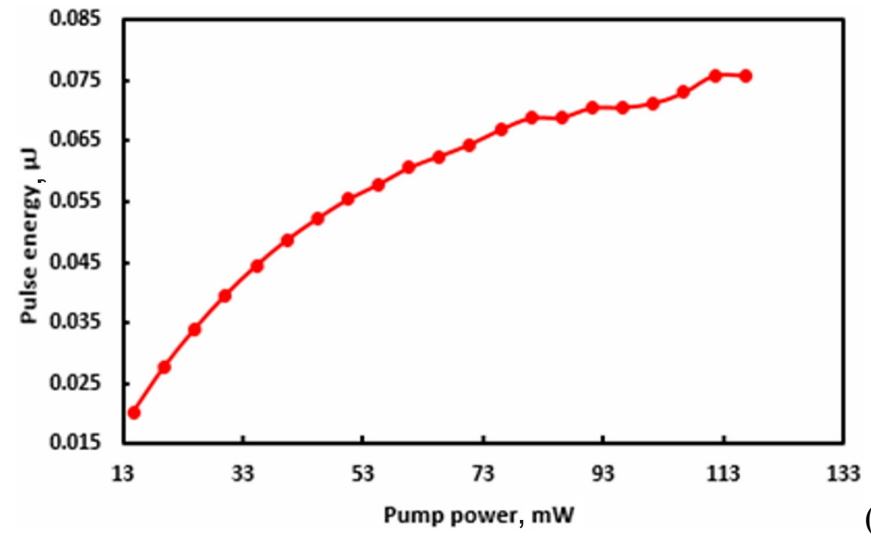

(b)

Fig. 3. Dependences of average output power (a) and pulse energy (b) on the pump power.

Fig. 4 shows dependences of the repetition rate and the pulse width (or the pulse duration) for our Q-switched EDFL as functions of the pump power. When the pump power increases from 14.85 to $116.61 \mathrm{~mW}$, the pulse repetition rate increases from 28.94 to $67.66 \mathrm{kHz}$. Under the same conditions, the pulse width decreases from 15.64 to $5.44 \mu \mathrm{s}$. 


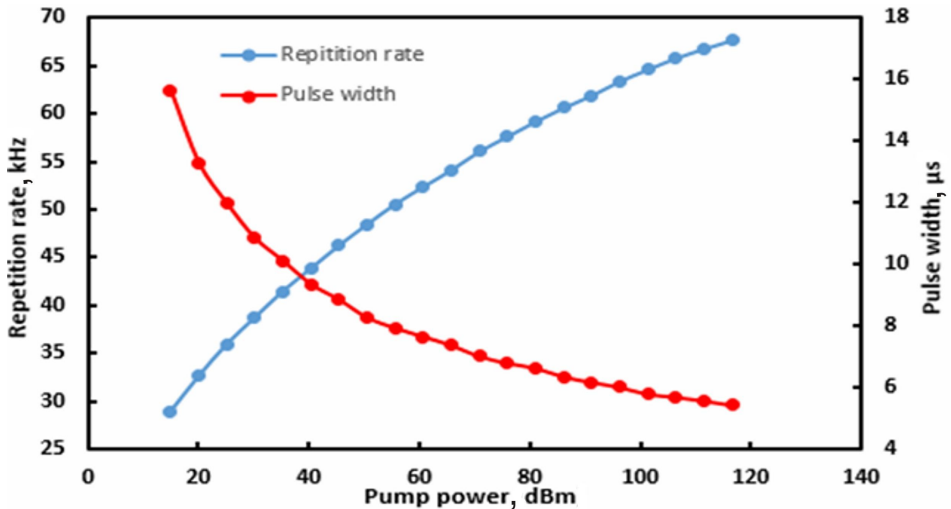

Fig. 4. Dependences of pulse repetition rate and pulse width on the pump power.

Fig. 5 demonstrates a train of laser pulses obtained from our Q-switched EDFL, using different pump powers and oscilloscope frequencies. The minimum and maximum pump powers in this experiment are the same as mentioned before.

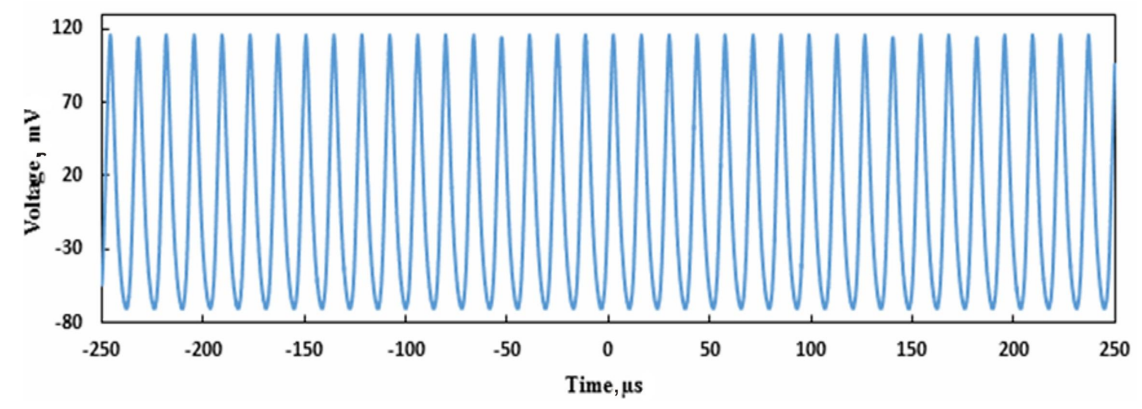

Fig. 5. Output pulse train of our MoS2-based Q-switched EDFL.

Fig. 6 shows the corresponding RF spectrum obtained at the maximum pump power and the repetition rate $67.66 \mathrm{kHz}$. Its main advantage is a possibility for checking and analyzing the noise in the laser system via calculations of signal-to-noise ratio. As in our previous experiments, the pump power in this experiment is being increased from 14.85 to $116.61 \mathrm{~mW}$.

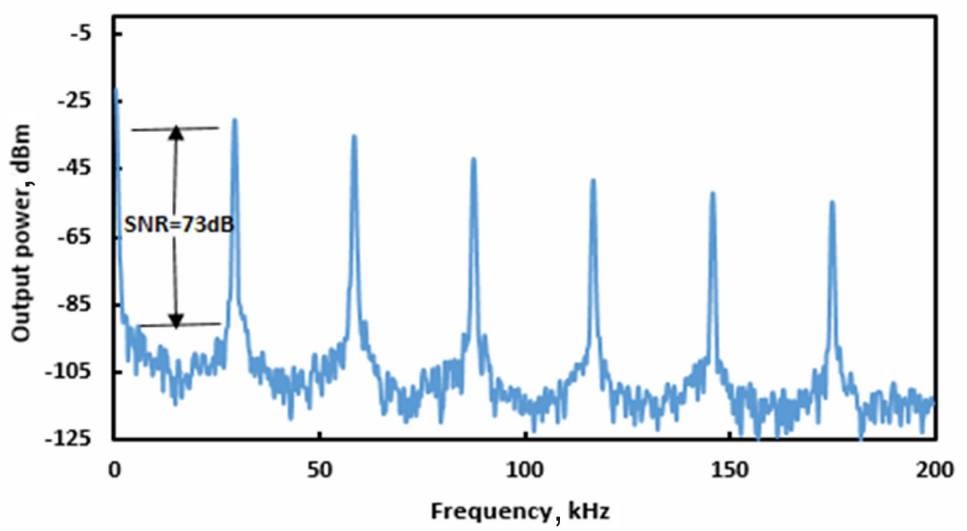

Fig. 6. RF spectrum observed at the pump power $116.61 \mathrm{~mW}$, using $67.66 \mathrm{kHz}$ spans. 


\subsection{Passively Q-switched fibre laser based on $W_{2}$}

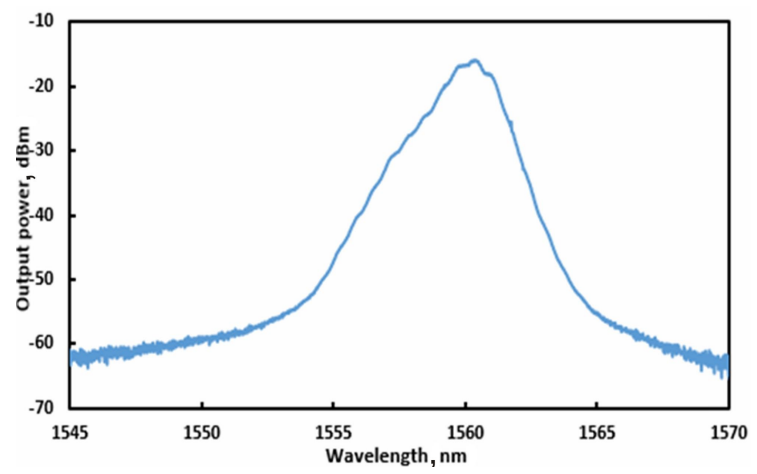

Fig. 7. Output spectrum of our Q-switched EDFL based on the $\mathrm{WS}_{2} \mathrm{SA}$

Fig. 7 illustrates the output spectrum achieved at the pump power $68.27 \mathrm{~mW}$ with our passively Q-switched EDFL based on the $\mathrm{WS}_{2} \mathrm{SA}$. Here the threshold peak at which the EDFL works corresponds to $1560.5 \mathrm{~nm}$.

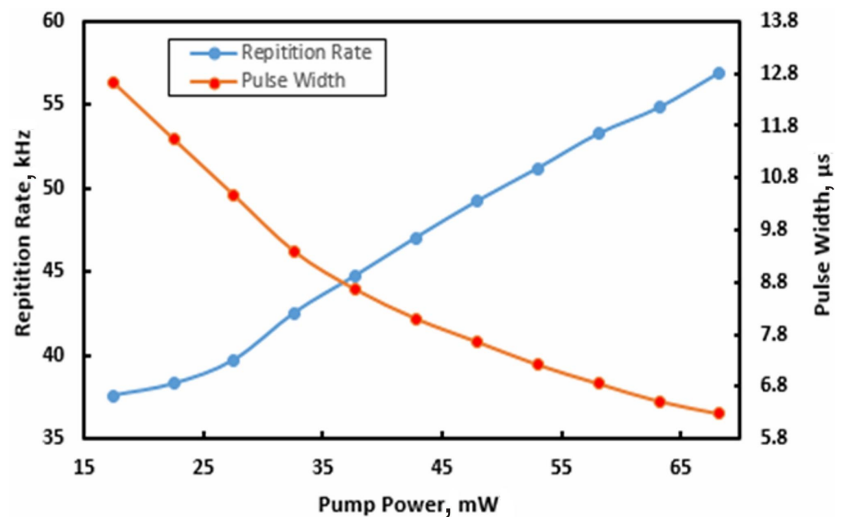

Fig. 8. Dependences of pulse repetition rate and pulse width on the pump power.

Fig. 8 shows how the pulse repetition rate and the pulse width of our Q-switched fibre laser depend on the pump power. The pulse repetition rate increases from 37.57 to $56.92 \mathrm{kHz}$ with increasing pump power. For each pump power and pulse repetition rate, no amplitude modulation in the pulse trains is observed and the Q-switched pulse output is stable. On the other hand, the pulse width decreases from $12.63 \mu \mathrm{s}$ near the pump threshold down to $6.28 \mu \mathrm{s}$ at the maximum pump power, $68.27 \mathrm{~mW}$. At the pump powers lower than $17.3 \mathrm{~mW}$, the pulse duration suddenly releases. The pulse width remains almost unchanged at higher pump powers $<68.27 \mathrm{~mW}$. This indicates clearly that the SA is saturated. Finally, the minimum pulse width detected in this experiment could, in principle, be still narrowed by shortening the cavity length and improving the modulation depth of the $\mathrm{WS}_{2} \mathrm{SA}$.

Fig. 9a shows the average output power of our passively Q-switched EDFL depending upon the pump power. Close to the maximal pumps, the average output power increases almost linearly with increasing input power. The maximum average output power is equal to $7.91 \mathrm{~mW}$ at the pump power $68.27 \mathrm{~mW}$, so that the slope efficiency can be estimated as $12 \%$. Fig. $9 \mathrm{~b}$ also shows the calculated pulse energy. Generally, this is done basing on the average output power and the repetition rate measured in the experiment. 

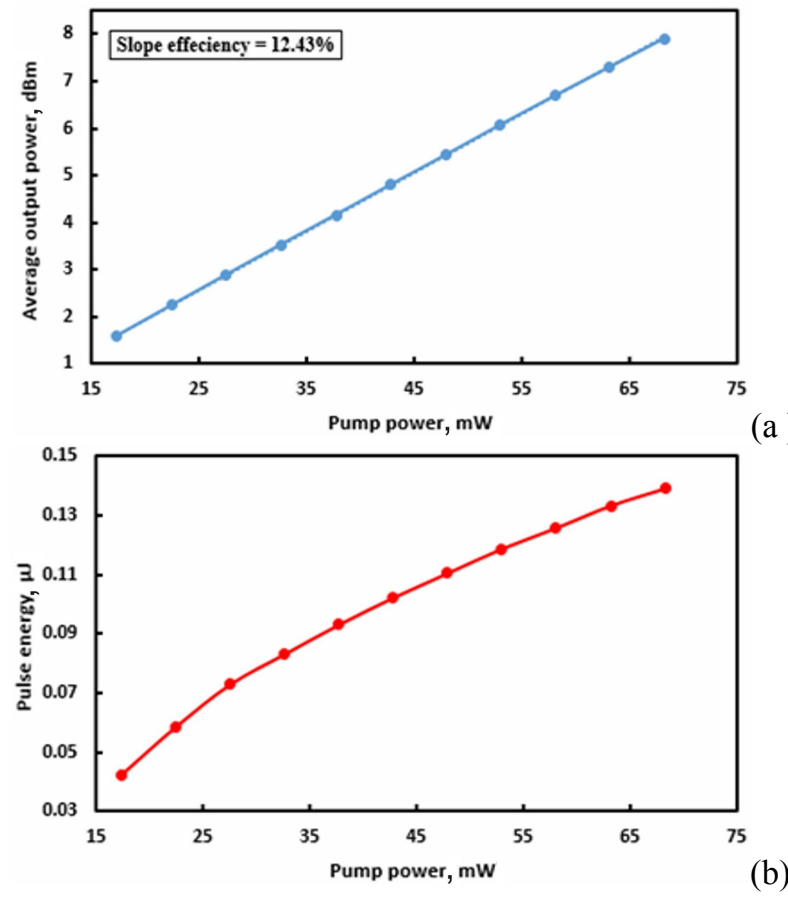

(a)

Fig. 9. Dependences of average output power (a) and the corresponding calculated pulse energy (b) on the pump power.

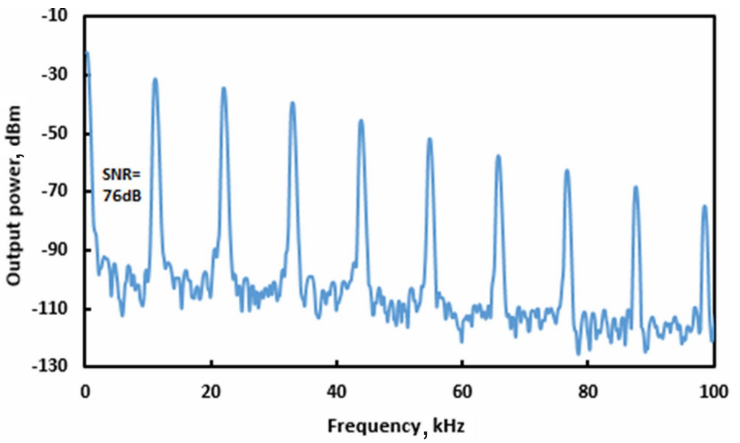

Fig. 10. RF spectrum observed with $\mathrm{WS}_{2} \mathrm{SA}$ at the pump power $68.27 \mathrm{~mW}$, using $56.92 \mathrm{kHz}$ spans.

Fig. 10 displays the RF spectrum corresponding to the Q-switched pulse, as detected at the pump power $68.27 \mathrm{~mW}$. The highest signal-to-noise ratio for this cavity is equal to $76 \mathrm{~dB}$ and the lowest one to $64 \mathrm{~dB}$. Issuing from low noises, one can see that the fibre laser operates in a highly stable Q-switching regime.

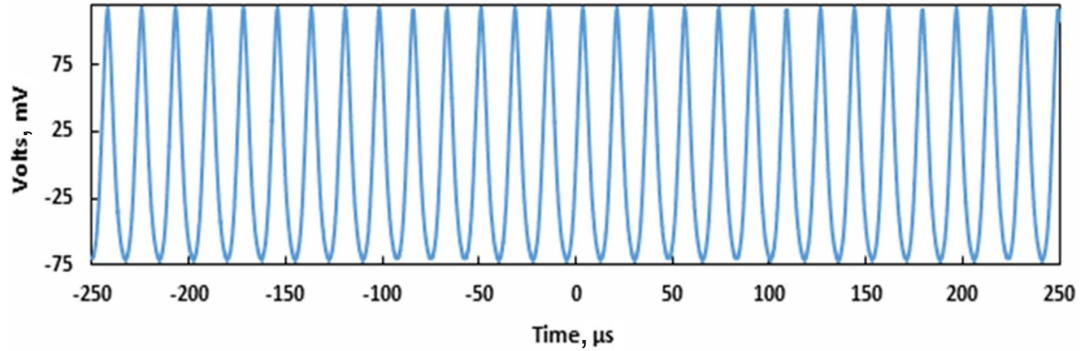

Fig. 11. Output pulse train observed for our Q-switched EDFL based on $\mathrm{WS}_{2}$. 
A stable work of the Q-switched EDFL is started at the pump power $17.3 \mathrm{~mW}$ and its output increases when the maximum pump power, $68.27 \mathrm{~mW}$, is reached. Fig. 11 illustrates typical oscilloscope traces observed for the Q-switched pulse trains at different pump powers and different repetition rates. The pulse repetition rate in our laser increases from 37.57 to $56.9 \mathrm{kHz}$ with increasing pump power. This is a typical feature of operation under the conditions of passive Q-switching.

\section{Conclusion}

We have developed a stable passively Q-switched EDFL based on $\mathrm{MoS}_{2}$ and $\mathrm{WS}_{2}$ as SAs. We have found that our Q-switched fibre laser based on the $\mathrm{WS}_{2} \mathrm{SA}$ generates more stable pulse trains when compared with the $\mathrm{MoS}_{2} \mathrm{SA}$. This makes $\mathrm{WS}_{2}$ a promising material for the pulsed-laser applications. The wavelength region in which the Q-switched pulses are obtained belongs to the so-called C-band region, with the central wavelengths $1559 \mathrm{~nm}$ or $1560 \mathrm{~nm}$ for the $\mathrm{MoS}_{2}-$ and $\mathrm{WS}_{2}$-based SA, respectively.

\section{Acknowledgment}

This work was supported financially by the IIUM under the grant RIGS (RIGS 15-148-0148). We also extend our thanks and gratitude to the Photonic Research Center for allowing us to do practical experiments at their laboratory.

\section{References}

1. Hongwei Chu, Shengzhi Zhao, Kejian Yang, Yufei Li, Dechun Li, Guiqiu Li, Jia Zhao, Wenchao Qiao, Tao Li, Yonggang Wang and Yishan Wang, 2015. Passively Q-switched Nd:GGG laser with a SWCNT as saturable absorber. Opt. Quant. Electron. 47: 697-703.

2. Ahmad H, Hassan N A, Aidit S N and Tiu Z C, 2016. Generation of tunable multi-wavelength EDFL by using graphene thin film as nonlinear medium and stabilizer. Opt. Laser Technol. 81: $67-69$.

3. Mears R, Reekie L, Jauncey I and Payne D, 1987. Low-noise erbium-doped fibre amplifier operating at $1.54 \mu \mathrm{m}$. Electron. Lett. 23: 1026-1028.

4. Bellemare A, Karbsek M, Riviere C, Babin F, Gang He, Roy V and Schinn G W, 2001. A broadly tunable erbium-doped fiber ring laser: experimentation and modeling. IEEE J. Select. Topics in Quant. Electron. 7: 22-29.

5. Ahmad H, Ruslan N E, Ismail M A, Reduan S A, Lee C S J, Sathiyan S, Sivabalan S and Harun S W, 2016. Passively Q-switched erbium-doped fiber laser at C-band region based on WS2 saturable absorber. Appl. Opt. 55: 1001-1005.

6. Saidin N, Ahmad F, Zen D I M, Hamida B A, Khan S, Ahmad H, Dimyati K and Harun S W, 2013. All-fiber graphene passively Q-switched nanosecond Thulium doped fiber laser at 1900 nm. In: IEEE International Conf. on Smart Instrumentation, Measurement and Appl., ICSIMA 2013. [6717968].

7. Pan L, Utkin I and Fedosejevs R, 2007. Passively Q-switched ytterbium-doped double-clad fiber laser with a Cr YAG saturable absorber. IEEE Photon. Technol. Lett. 19: 1979-1981.

8. Xuekun Bai, Jun Yuan, Shaofei Wang, Dengfeng Fan, Jie Gu, Yi Huang, Yunhe Zhao, Qianwu Zhang, Shengli Pu and Xianglong Zeng, Passively Q-switching of erbium-doped fiber laser using ferrite as saturable absorber. In: 2015 Conf. on Lasers and Electro-Optics. Pacific Rim (Optical Society of America, 2015), paper 26C1_4. 
Mohamed K.H., Hamida B.A., Sheroz Khan, Hussein L.A., Ahmat M.O., El Ismail, Kadir N.A.A., Latif A.A. and Harun S.W. 2017. Q-switched erbium-doped fibre laser based on molybdenum disulfide and tungsten disulfide as saturable absorbers. Ukr.J.Phys.Opt. 18: 20 - 27

Анотація. Експериментально реалізовано ербієвий волоконний лазер 3 пасивною модуляцією добротності, який використовує дисульфід молібдену $\left(\mathrm{MoS}_{2}\right)$ i дисульфід вольфраму $\left(W_{2}\right)$ як насичені поглиначі. Результати засвідчують, щуо побудований на $\mathrm{WS}_{2}$ волоконний лазер генерує стабільнімі послідовності імпульсів, порівняно з випадком насиченого поглинача на основі $\mathrm{MoS}_{2}$. Діапазон довжин хвиль, у якому було отримано 3 модульовані імпульси, відповідає так званій С-смузі. Центральні довжини хвиль для поглиначів на $\mathrm{MoS}_{2}$ i $\mathrm{WS}_{2}$ дорівнюють відповідно 1559 і 1560 нм. 